\title{
Sífilis secundária em gestante: relato de caso
}

\author{
Syphilis secondary in pregnancy: case report \\ La sífilis secundaria durante el embarazo: reporte de caso \\ Bruna da Fonseca WASTNER \\ Rafaela Savio MELZER \\ Tuanny Carvalho de Lima do NASCIMENTO \\ Guilherme KLEIN PARISE \\ Roberta Targa STRAMANDINOLI-ZANICOTTI \\ Laurindo Moacir SASSI
}

Department of Oral and Maxillofacial Surgery, Erasto Gaertner Hospital, Curitiba, Paraná, Brazil.

\begin{abstract}
Resumo
Sífilis é uma doença sexualmente transmissível causada por bactérias gram-negativas do grupo das espiroquetas, Treponema pallidum. Ela pode ser congênita ou adquirida. Seus sinais e sintomas podem variar dependendo do seu estádio. Foi relatado o caso clínico de uma paciente de 25 anos que ao exame físico apresentou múltiplas placas brancas, rugosas em mucosa bucal e orofaringe, com diagnóstico após exames laboratoriais e anatomopatológico de sífilis secundária. Paciente foi encaminhada ao Serviço de Infectologia, para tratamento clínico com penicilina G benzatina. Para descartar a existência de gravidez, foi solicitado exame BetaHCG, o qual foi negativo. A paciente foi orientada sobre educação sexual, DSTs e a evitar gravidez até liberação pelo médico infectologista, devido ao risco de o bebê ser contaminado. Porém, antes de iniciar o tratamento, enquanto aguardava a liberação da medicação, a paciente engravidou. No seu retorno com a equipe odontológica, informou aos profissionais que estava gestante de 11 semanas. Clinicamente apresentou remissão total das lesões orais. Conclui-se que, apesar de muito ser discutido acerca da necessidade de novos testes diagnósticos que sejam mais práticos, rápidos e acessíveis para facilitar a detecção da doença, torna-se essencial educar a população desde a época escolar sobre as DSTs.
\end{abstract}

Descritores: Sífilis; Boca; Gravidez.

\begin{abstract}
Syphilis is a sexually transmitted disease caused by gram-negative bacteria of the spirochete group, Treponema pallidum. It may be congenital or acquired. Your signs and symptoms may vary depending on your stage. The clinical case of a 25-year-old female patient who on physical examination presented multiple white plaques, wrinkled in the buccal mucosa and oropharynx, with diagnosis after laboratory and anatomopathological examination of secondary syphilis. Patient was referred to the Infectology Service, for clinical treatment with benzathine penicillin G. To rule out the existence of pregnancy, Beta-HCG examination was requested, which was negative. The patient received instruction about sex education, STIs and to avoid pregnancy until released by the infectious disease physician due to the risk of the baby being contaminated. However, before beginning treatment, while awaiting the release of the medication, the patient became pregnant. On her return with the dental team, she informed the professionals that she was pregnant for 11 weeks. Clinically, the patient presented total remission of oral lesions. It is concluded that, although much is discussed about the need for new diagnostic tests that are more practical, fast and accessible to facilitate the detection of the disease, it is essential to educate the population from school time about STDs.

Descriptors: Syphilis; Mouth; Pregnancy.
\end{abstract}

\section{Resumen}

La sífilis es una enfermedad de transmisión sexual causada por bacterias gram-negativas del grupo de espiroquetas, Treponema pallidum. Puede ser congénita o adquirida. Sus signos y síntomas pueden variar dependiendo de su estadio. Se informará el caso clínico de una paciente de 25 años que al examen físico presentó múltiples placas blancas, rugosas en mucosa bucal y orofaringe, con diagnóstico después de exámenes de laboratorio y anatomopatológico de sífilis secundaria. El paciente fue encaminado al Servicio de Infectología, para tratamiento clínico con penicilina G benzatina. Para descartar la existencia de embarazo, se pidió el examen betahcg, el cual fue negativo. La paciente fue orientada sobre educación sexual, ETS y evitar el embarazo hasta la liberación por el médico infectologista, debido al riesgo de que el bebé sea contaminado. Sin embargo, antes de iniciar el tratamiento, mientras esperaba la liberación de la medicación, la paciente se embarazó. A su regreso con el equipo odontológico, informó a los profesionales que estaba embarazada de 11 semanas. Clínicamente presentó remisión total de las lesiones orales. Se concluye que, aunque se discute mucho sobre la necesidad de nuevas pruebas diagnósticas que sean más prácticas, rápidas y accesibles para facilitar la detección de la enfermedad, es esencial educar a la población desde la época escolar sobre las ETS.

Descriptores: Sífilis; Boca; Embarazo.

\section{INTRODUÇÃO}

A sífilis é uma doença sexualmente transmissível causada por bactérias gram-negativas do grupo das espiroquetas, Treponema pallidum. A manifestação desta doença é um reflexo da diminuição do uso de métodos contraceptivos, número elevado de parceiros sexuais e realização de sexo oral sem proteção ${ }^{1}$.

Existem dois tipos de sífilis: a congênita e a adquirida. O primeiro tipo é transmitido verticalmente pela disseminação transplacentária, ao passo que a adquirida, é sexualmente transmitida ${ }^{1}$.

A doença adquirida pode ser classificada em quatro estágios: primário, secundário, latente e terciário. As lesões orais são, principalmente, associadas à sífilis secundária, embora todas as etapas possam apresentar manifestações bucais $^{2}$. A sífilis também pode ocorrer como coinfecção em pacientes HIV positivos ${ }^{1}$.
O primeiro estágio da doença é caracterizado por um cancro que manifesta aproximadamente 90 dias após a exposição à bactéria e apresenta remissão espontânea entre duas e oito semanas. O estágio secundário ocorre entre a segunda e décima segunda semana após exposição quando lesões afetam mucosas e peles, podendo ou não diminuir o aparecimento de lesões sem tratamento e entrando em fase de latência. Por fim, o estágio terciário, também chamado de fase tardia, apresentam neurossífilis que emergem três ou mais anos após contato com o Treponema pallidum ${ }^{3}$.

As lesões orais de sífilis são clinicamente heterogêneas e incluem máculas, pápulas, placas e úlceras que podem ser únicas ou múltiplas, podendo ou não estar associadas à faringite, amigdalite ou laringite, com presença de linfonodos palpáveis ${ }^{4}$. 
A Sífilis primária é caracterizada pelo cancro, uma lesão ulcerada de bordas elevadas, endurecidas, indolor, que pode medir entre um a dois centímetros, e frequentemente é solitária. Esta lesão surge no local da inoculação do Treponema pallidum, sendo evidente clinicamente entre 3 a 90 dias. Na região intrabucal as áreas mais acometidas são: lábios, língua, palato, gengiva e amígdalas. Pode haver remissão espontaneamente da lesão entre 3 e 6 semanas ${ }^{5-8}$.

A Sífilis secundária se manifesta entre 4 a 6 semanas após a lesão primária. Porém alguns pacientes não relatam histórico prévio ao cancro ${ }^{6}$. Na região intrabucal há presença de placas esbranquiçadas, irregulares, indolores, podendo ser destacáveis ${ }^{7,9}$. Acomete com maior frequência regiões de língua, lábios, mucosa jugal, palato e amígdalas. Sua resolução espontânea geralmente ocorre entre 3 e 12 semanas $^{5,8}$

Histologicamente as características podem ser muito indicativas de sífilis: presença de inflamação crônica com presença de infiltrado de células plasmáticas perivascular com líquido liquenóide, aparência de banda, hiperplasia epitelial, micro-abscessos no epitélio ou neurite ${ }^{10}$.

O primeiro tratamento efetivo para esta doença foi realizado com penicilina, em 1943 e, desde então, é a droga de escolha para a sífilis (penicilina $G$ benzatina ou penicilina $G$ procaína $)^{4}$.

O presente trabalho tem como objetivo relatar o caso de sífilis secundária acometendo uma jovem gestante de 25 anos, a qual foi diagnosticada no Serviço de Cirurgia e Traumatologia Bucomaxilofacial do Hospital Erasto Gaertner.

\section{CASO CLÍNICO}

Paciente feminino, 25 anos, feoderma, foi encaminhada ao Serviço de Cirurgia Bucomaxilofacial do Hospital Erasto Gaertner/Curitiba-PR relatando "bolinhas na boca e garganta" que surgiram há 3 meses, que doíam durante fala e deglutição. A paciente negou hábitos nocivos à saúde como tabagismo e etilismo, bem como alergias e uso contínuo de medicamentos.

Questionada, afirmou ser mãe de uma criança de 2 anos de idade. Ainda sobre a história social, mencionou não ter mais contato com o pai da criança e definiu-se como sexualmente inativa no momento da consulta inicial. Ao exame intrabucal, observou-se múltiplas placas brancas, de superfície rugosa, não-sangrantes e não-removíveis, em comissura labial bilateral, orofaringe e ventre lingual (Figura 1).

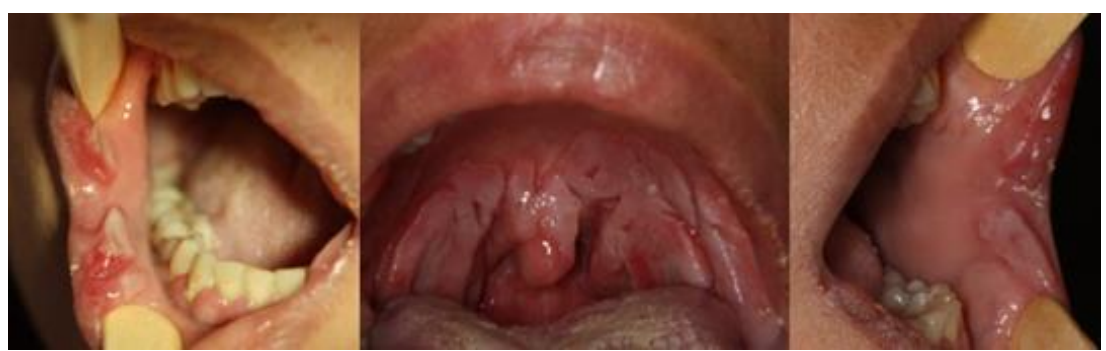

Figura 1: Aspecto inicial da paciente, mostrando as placas sifilíticas em mucosa bucal.

A paciente também apresentava nódulo de aproximadamente $2 \mathrm{~mm}$ na esclera do olho esquerdo. A hipótese diagnóstica clínica inicial foi de placas sifilíticas. Exames laboratoriais específicos para sífilis foram solicitados com resultados positivos (VDRL e FTA ABS), sendo não reagente para outras sorologias (HIV, Hepatites). Foi realizada uma biópsia incisional da mucosa jugal direita, do ventre lingual $\mathrm{e}$ do palato mole. $\mathrm{O}$ anatomopatológico mostrou mucosa ulcerada com denso infiltrado inflamatório crônico, difuso em córion, rico em plasmócitos, definindo um quadro compatível com a hipótese de sífilis. A paciente foi encaminhada ao Serviço de Infectologia da mesma instituição, que realizou o tratamento clínico com penicilina $G$ benzatina. Para descartar a existência de gravidez, foi solicitado exame Beta-HCG, o qual foi negativo. A paciente foi orientada sobre educação sexual e a evitar gravidez até liberação pelo médico infectologista, devido ao risco de o bebê ser contaminado. A paciente enfrentou dificuldades em conseguir a medicação prescrita na Unidade Básica de Saúde, o que atrasou o início do tratamento. Após o término da medicação, a paciente retornou sem sinais clínicos da doença (Figura 2). Porém, informou aos profissionais que estava gestante de 11 semanas, sendo que engravidou no período que estava aguardando a liberação do antibiótico.

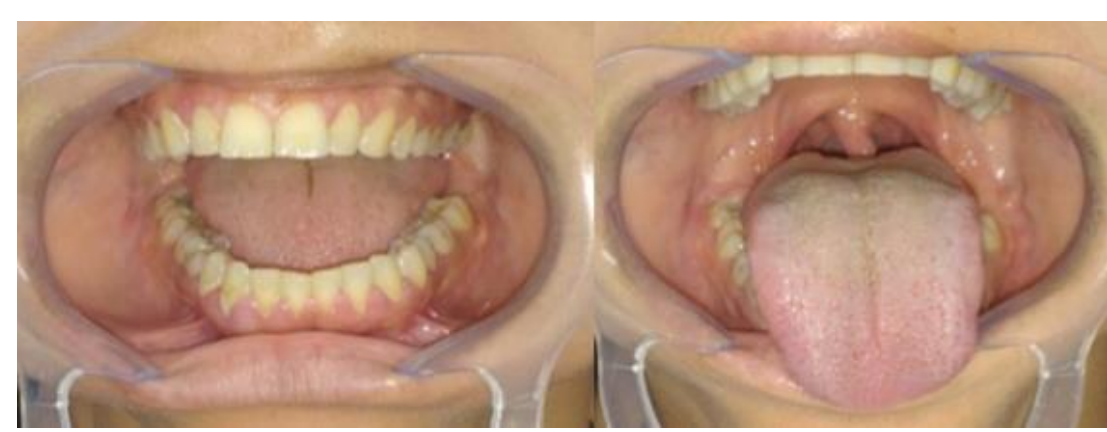

Figura 2: Aspecto intrabucal da paciente após o tratamento evidenciando a remissão total das lesões.

\section{DISCUSSÃO}

De acordo com a Constituição Federal, Seção II, Art. 196, "A saúde é direito de todos e dever do Estado, garantido mediante políticas sociais e econômicas que visem à redução do risco de doença e de outros agravos e ao acesso universal e igualitário às ações e serviços para sua promoção, proteção e recuperação" ${ }^{11}$. No Brasil, em 19 de setembro de 1990, a lei número 8.080 foi sancionada, regulando, em todo território nacional, as ações e serviços de saúde, dando início, assim, ao Sistema Único de Saúde $(\mathrm{SUS})^{12}$. Nos anos seguintes, foram propostas diversas mudanças no funcionamento dos serviços de saúde, baseado nas experiências de outros países como Canadá, Cuba, Suécia e Inglaterra, que já possuíam um sistema direcionado ao cuidado da família ${ }^{13}$. Assim, em 1993, surge o Programa de Saúde da Família. Em 2011, o programa foi consolidado como política e teve seu nome alterado para Estratégia da Saúde da Família $(\mathrm{ESF})^{14}$.

Desenvolvida pelo Ministério da Saúde (MS), a Política Nacional de Atenção Integral à Saúde da Mulher é composta de ações em diversas áreas, como prevenção e tratamento das principais condições que afetam esse grupo, além de informar sobre sexualidade. A adesão a este programa específico é prevista no orçamento dos municípios quando do repasse de verba de saúde, sendo que, a decisão do uso do recurso fica a cargo do gestor municipal ${ }^{15}$. A rede de serviços do SUS de Curitiba é uma das referências em qualidade no país, contando com 65 ESF e 6 Centros de Especialidade, sendo um deles a Mãe Curitiba, voltada ao atendimento integral das gestantes ${ }^{16}$.

Uma das preocupações quando se trata gestantes é, sem dúvidas, as doenças sexualmente transmissíveis (DSTs), que podem ser transmitidas ao feto durante a gestação ou parto, como toxoplasmose, rubéola, HIV, hepatite B e sífilis. ${ }^{9}$ A sífilis na gestação, a doença abordada nesse artigo especificamente, ocasiona mais de 300 mil mortes de fetos e recém-natos por ano no mundo, além de aumentar o risco de aborto. No Brasil, desde o ano de 2005, 
uma Portaria determinou a obrigatoriedade de notificar as gestantes com sífilis, o que levou a um visível aumento no número de casos conhecidos ${ }^{17}$.

O tratamento de escolha para essa doença é o medicamentoso, através da utilização de penicilina benzatina. Porém, desde o ano de 2014, o Brasil e outros países vêm enfrentando dificuldade no recebimento do antibiótico, devido escassez da matéria-prima ${ }^{17}$. Outros medicamentos que podem ser utilizados são a doxaciclina, tetraciclina e eritromicina, porém, todos estes são contraindicados para uso em gestantes ${ }^{18}$.

Dados do Ministério da Saúde referentes ao ano de 2016 mostram um aumento de casos registrados de sífilis em gestantes, passando de 39.789 em 2015 para 129.757 casos em 2016. E relação à sífilis congênita, os dados apontam 36.000 casos em 2015 e 79.670 em 2016. Ressaltase que tais dados não representam puramente um aumento na incidência da doença, e sim uma melhora nos programas de vigilância em saúde que puderam diagnosticar mais casos. Dos casos de sífilis adquirida no período de 2010 a 2016, constatou-se que $20,5 \%$ destes foram de pessoas residentes na região Sul do Brasil. Em relação à sífilis em gestantes, observou-se em 2015 que 51,6\% das grávidas infectadas tinham entre 20 e 29 anos de idade, com primeiro grau escolar incompleto em 20,9\%. Além disso, em 2015, ressalta-se que a maioria dos casos notificados foi de sífilis primária $(31,6 \%)$, seguido da forma latente $(23,6 \%)$, terciária $(10,7 \%)$ e, por último, a secundária $(5,8 \%)^{17}$. Estas informações são compatíveis com o caso apresentado nesse artigo, que trata de uma paciente de 25 anos, com baixo nível de escolaridade e que não possuía informação alguma sobre sífilis, sua prevenção e possíveis complicações. $\mathrm{Na}$ experiência do Serviço de Cirurgia Bucomaxilofacial do Hospital Erasto Gaertner, que atua no diagnóstico e tratamento de lesões de boca há 28 anos, nunca foi diagnosticado um caso de sífilis secundária em boca. Apenas nos 3 primeiros meses de 2017, já foram diagnosticados mais dois novos casos de sífilis com lesões bucais, o que condiz com o aumento do número de pacientes conforme documento pelo Ministério de Saúde.

Nos casos em que a mãe é infectada antes ou no início da gestação e não recebe tratamento adequado, estima-se que aproximadamente 80 a $100 \%$ dos casos acarretem em contaminação do feto. Por outro lado, nos casos em que a contaminação se dá mais tardiamente na gestação e não é realizado o tratamento correto, a infecção fetal ocorre em aproximadamente $30 \%$ dos casos. As complicações mais comuns são: abortamento espontâneo, morte do recém-nato, parto prematuro e comprometimento oftalmológico/auditivo/neurológico da criança ${ }^{19}$.

\section{CONSIDERAÇÕES FINAIS}

Apesar de muito ser discutido acerca da necessidade de novos testes diagnósticos que sejam mais práticos, rápidos e acessíveis para facilitar a detecção da doença, acreditamos que o essencial é educar a população desde a época escolar sobre as DSTs, ensinar sua prevenção e orientar o tratamento e as possíveis complicações. Esse trabalho deve ser constante e exaustivo, a fim de impactar realmente as pessoas, não apenas a curto como também a longo prazo, objetivando adultos responsáveis com a saúde na sexualidade e na gestação. Somente com ações desse tipo, podemos esperar por mudanças reais. Não se deve culpar o Sistema de Saúde por um problema que o extrapola. A deficiência existe no Sistema de Ensino, na base familiar e em outros vários setores da sociedade em que esse indivíduo se insere.

\section{REFERÊNCIAS}

1. Leuci S, Martina S, Adamo D, Ruoppo E, Santarelli A, Sorrentino $\mathrm{R}$ et al. Oral syphilis: a retrospective analysis of 12 cases and a review of the literature. Oral Diseases. 2013; 19(8):738-46.

2. de Paulo LF, Servato JP, Oliveira MT, Duriguetto AF $\mathrm{Jr}$, Zanetta-Barbosa D. Oral manifestations of secondary syphilis. Int J Infect Dis. 2015; 35:40-2.

3. Seibt CE, Munerato MC. Secondary syphilis in the oral cavity and the role of the dental surgeon in STD prevention, diagnosis and treatment: a case series study. Braz J Infect Dis. 2016; 20(4):393-8.

4. Plana-Pla A, Pelegrín-Colás L, Bielsa-Marsol I, Ferrandiz-Foraster C. Secondary syphilis presenting as oral lesions and posterior placoid chorioretinitis in an immunocompetent patient. Actas Dermosifiliogr. 2016;107:783-84.

5. Barrett AW, VillarroeI Dorrego M, Hodgson TA, Porter SR, Hopper C, Argiriadou AS et al. The histopathology of syphilis of the oral mucosa. J Oral Pathol Med. 2004; 33(5): 286-91.

6. Little JW. Gonorrhea: an update. Oral Surg Oral Med Oral Pathol Oral Radiol Endod. 2005; 101(2):137-43.

7. Neville BW, Damm DD, Allen, CM. Patologia Oral e Maxilofacial. 2. ed. Rio de Janeiro: Guanabara Koogan; 2004.

8. Valente $T$, Scalercio $M$, Israel $M$, Ramos ME. Diagnóstico da sífilis a partir das manifestações bucais. Rev bras odontol. 2008; 65(2):159-64.

9. Bruce AJ, Rogers RS 3rd. Oral manifestations of sexually transmitted diseases. Clin Dermatol. 2004; 22(6):520-7.

10. Siqueira CS, Saturno JL, de Sousa SC, de Silveira FR. Diagnostic approaches in unsuspected oral lesions of syphilis. Int J Oral Maxillofac Surg. 2014; 43(12): 1436-40.

11. Brasil, Constituição (1988). Constituição da República Federativa do Brasil. Brasília, DF: Senado Federal:Centro Gráfico; 1988.

12. Brasil. Lei No 8080/90, de 19 de setembro de 1990 . Brasília: TF; 1990. Disponível em https://www. planalto.gov.br/ccivil_03/LEIS/L8080.htm

13. Vianna ALA, Dal Poz MR. Estudo sobre o processo de reforma em saúde no Brasil. (RJ): Abril; 1998.

14. Ministério da Saúde (BR), Secretaria de Atenção à Saúde, Departamento de Atenção Básica. Política Nacional de Atenção Básica (PNAB). Brasília: Ministério da Saúde; 2012.

15. Brasil, Ministério da Saúde. O SUS de A a Z : garantindo saúde nos municípios / Ministério da Saúde, Conselho Nacional das Secretarias Municipais de Saúde. - 3. ed. Brasília: Editora do Ministério da Saúde; 2009.

16. Prefeitura Municipal de Curitiba. Rede de Serviços do SUS Curitiba. Disponível em http://www.saude. curitiba.pr.gov.br/a-secretaria/rede-de-atencao.html

17. Secretaria de Vigilância em Saúde. Boletim Epidemiológico - Sífilis 2016. Ministério da Saúde. 2016; 47(35):1-32.

18. Departamento de DST, AIDS e Hepatites virais. Secretaria de Vigilância em Saúde. Ministério da Saúde. Prevenção e atenção das infecções sexualmente 
transmissíveis (IST). Excerto do manual de bolso sífilis.

19. Magalhães DMS, Kawaguchi IAL, Dias A, Calderon IMP. A sífilis na gestação e sua influência na morbimortalidade materno-infantil. Com Ciências Saúde 2011; 22(1):S43-54.

\section{CONFLITO DE INTERESSES}

Os autores declaram não haver conflitos de interesse.

\section{AUTOR PARA CORRESPONDÊNCIA}

Bruna da Fonseca Wastner

bru.wastner@hotmail.com

Submetido em 24/06/2017 Aceito em 10/08/2017 